\title{
Interval Solution for Nonlinear Programming of Maximizing the Fatigue Life of V-Belt under Polymorphic Uncertain Environment
}

\author{
Zhong Wan, ${ }^{1}$ ShaoJun Zhang, ${ }^{1,2}$ and Yanju Zhou ${ }^{3}$ \\ ${ }^{1}$ School of Mathematics and Statistics, Central South University, Changsha 410083, China \\ ${ }^{2}$ School of Mechanical Engineering, Hunan University of Technology, ZhuZhou 412007, China \\ ${ }^{3}$ School of Business, Central South University, Changsha 410083, China \\ Correspondence should be addressed to Zhong Wan; wanmath@163.com
}

Received 22 January 2013; Revised 13 March 2013; Accepted 13 March 2013

Academic Editor: Alexander P. Seyranian

Copyright ( 2013 Zhong Wan et al. This is an open access article distributed under the Creative Commons Attribution License, which permits unrestricted use, distribution, and reproduction in any medium, provided the original work is properly cited.

\begin{abstract}
In accord with the practical engineering design conditions, a nonlinear programming model is constructed for maximizing the fatigue life of V-belt drive in which some polymorphic uncertainties are incorporated. For a given satisfaction level and a confidence level, an equivalent formulation of this uncertain optimization model is obtained where only interval parameters are involved. Based on the concepts of maximal and minimal range inequalities for describing interval inequality, the interval parameter model is decomposed into two standard nonlinear programming problems, and an algorithm, called two-step based sampling algorithm, is developed to find an interval optimal solution for the original problem. Case study is employed to demonstrate the validity and practicability of the constructed model and the algorithm.
\end{abstract}

\section{Introduction}

It is well known that V-belt driving is widely used in all kinds of machines. Some deterministic models have been established to optimize the volume or the driving capacity of a V-belt drive system (see, e.g., [1, 2]). In [3], the effect of angular speed on the behavior of V-belt drive system is studied by finite element method. In [4], a deterministic model is constructed to identify the contact parameters between belt and pulley based on experimental data. In [1], a global optimization algorithm, called optimal line segment algorithm, is developed to find a global maximizer of the deterministic model for maximizing the carrying power of V-belt drive. In addition, in [5], a deterministic model is constructed to maximize the fatigue life of V-belt and a global optimal solution is found by the algorithm developed in [1].

Nevertheless, the design problem in V-belt drive is often subject to a variety of uncertainties. For example, the bending modulus $E_{b}$, of elasticity, the carrying power $P$, of a single V-belt, the rotational speed $n_{1}$, of small pulley and the experimental constant $C$ employed to calculate the rating power of V-belt, are often affected by the uncertain nature of various factors such as the raw materials, the manufacturing techniques and the environmental conditions. The ratio of transmission $i$ is hard to be determined exactly since it is affected mainly by the sliding ratio of elasticity. Thus, only an approximate range of values for $i$ is given in practice. In addition, some limitations on the decision variables and the constraints have transient features, hence, it is unsuitable to fix these values in the model of designing the V-belt drive system. It is clear that a deterministic mathematical model is unlikely to lead to a satisfactory solution for such a problem. Actually, as a constructed model in this paper, the optimal design problem of V-belt drive system is a polymorphic uncertain nonlinear program (PUNP) (also see [6]).

The existent uncertain programming methods such as stochastic programming (see, e.g., [7]), fuzzy programming (see, e.g., [8]) and interval programming (see, e.g., [9]) can not solve the PUNP problem directly. It is noted that there are many contributions which deal with the simple 
polymorphic uncertain linear program (PULP). For example, in [10], the multi-objective linear programming problem containing fuzzy random parameters is solved by fuzzy random simulation. In [11], a class of mix design linear programming problems involved with interval and random coefficients is studied to find an optimal mixture scheme. In [12], a hybrid two-stage stochastic optimization model is constructed for the regional energy systems planning which is associated with interval, fuzzy and random parameters.

There are also studies on nonlinear optimization model under polymorphic uncertainties. In [13], a nonlinear optimization model for stream water quality management including interval and fuzzy parameters is solved by the method of piecewise linearization. In [14] about the problem of random vibrations of structures, the probabilistic characteristics of the excitation are assumed to be given as depending on some parameters which are not known in advance. But it is known that these parameters belong to a bounded, convex set. This problem is dealt with as an anti-optimization problem of finding the least favorable values of the mean-square response. In [15], a method of Reliability-Based Design with the mixture of random and interval variables is provided. The reliability is considered under the condition of the worst combination of interval variables. With this treatment, the problem is conducted within a series of cycles of deterministic optimization and reliability analysis. The author also makes sensitivity analysis in terms of intervals for the above problem in $[16]$. In $[17,18]$, a reliability analysis problem with the mixture of random and interval variables is also discussed. In its model some key parameters in the random distribution functions are given variation intervals instead of precise values. Two kinds of hybrid reliability models are constructed based on the reliability index approach and the performance measurement approach. Based on the monotonicity analysis, two efficient algorithms are formulated to solve the above two models, respectively, in which the outer-layer optimization in terms of random variables and the inner-layer optimization in terms of interval parameters are executed by turns. In [6], a new concept of polymorphic uncertain system is introduced, where a prediction method, called the piecewise inference algorithm, is developed for estimating a parameter with polymorphic uncertainty. It is then shown that this method is promising for the forecasting of the components of alkali in the sintering process of aluminium.

Very recently in [19], a robust optimization method is developed to optimize the nonlinear problem of maximizing the $V$-belt's fatigue life with polymorphic uncertainties based on the concept of possibility degree for the order of two interval numbers. By the approach in [19], a deterministic optimal design scheme is obtained for a given satisfaction level. It is also noted that, in [20], a two-step based sampling method is developed to solve the nonlinear problem of maximizing the power transmission capacity in a system of $\mathrm{V}$-belt driving with polymorphic uncertainties and an interval solution for the original model is obtained for a given satisfaction level. It is clear that a deterministic optimal design scheme and an interval optimal solution have their individual significance in engineering application.
Different from the existent results in $[5,6,19,20]$, this paper intends to construct a PUNP model to formulate a problem of maximizing the fatigue life of a V-belt system with polymorphic uncertainty, which turns out to be a nonlinear uncertain programming model. By fixing a satisfaction level which is used to determine the cut set of a fuzzy number and a confidence level, an equivalent formulation of this uncertain optimization model will be obtained where only interval parameters are involved. Based on the concepts of maximal and minimal range inequalities for describing interval inequality, the interval parameter model is decomposed into two standard nonlinear programming problems. Then, we will develop an algorithm, called two-step based sampling algorithm, to find an interval optimal solution for the original problem. Case study is employed to demonstrate the validity and practicability of the constructed model and the algorithm.

The rest of this paper is organized as follows. In next section, a PUNP model is construct to formulate a problem of maximizing the fatigue life of a V-belt system under some assumptions. In Section 3, an equivalent formulation of this uncertain optimization model is presented. A solution method of the model is studied in Section 4. Section 5 is devoted to case study. Final remarks will be given in the last section.

\section{PUNP Model for Maximizing Fatigue Life of V-Belt}

In this section, a PUNP model for the problem of maximizing the fatigue life of V-belt will be constructed in an uncertain environment.

Firstly, we shall present a deterministic model to maximize the $\mathrm{V}$-belt's fatigue life.

Let $x_{1}, x_{2}$, and $x_{3}$ be the length $(\mathrm{mm})$ of $\mathrm{V}$-belt, the diameter $(\mathrm{mm})$ of small pulley, and the wrap angle ( $\mathrm{rad}$ ) of $\mathrm{V}$-belt on the small pulley, respectively. Denote $\mathbf{x}=$ $\left(x_{1}, x_{2}, x_{3}\right)^{T}$. For a known type of $\mathrm{V}$-belt, if the rotational speed $n_{1}(\mathrm{r} / \mathrm{min})$ of small pulley, the carrying power $P(\mathrm{~kW})$ of a single V-belt, and the transmission ratio $i$ are fixed, then the deterministic model of maximizing the fatigue life of $\mathrm{V}$ belt can be constructed as follows (see [19]):

$$
\begin{aligned}
\min W(\mathbf{x})= & C_{1} C^{-1 / m} n_{1}^{-(m-1) / m} x_{1}^{-1 / m} x_{2}^{-(m-1) / m} \\
& \times\left[C_{2} P\left(1-e^{-C_{5} x_{3}}\right)^{-1}+C_{3} n_{1} E_{b}+C_{4} n_{1}^{3} x_{2}^{3}\right]
\end{aligned}
$$

s.t. $\quad 8 C_{6}(i+1) x_{2}$

$$
\begin{aligned}
& -\left(2 x_{1}-\pi(i+1) x_{2}\right. \\
& \left.+\sqrt{\left[2 x_{1}-\pi(i+1) x_{2}\right]^{2}-8(i-1)^{2} x_{2}^{2}}\right) \leq 0, \\
& \left(2 x_{1}-\pi(i+1) x_{2}+\sqrt{\left[2 x_{1}-\pi(i+1) x_{2}\right]^{2}-8(i-1)^{2} x_{2}^{2}}\right) \\
& -8 C_{7}(i+1) x_{2} \leq 0,
\end{aligned}
$$




$$
\begin{aligned}
& x_{3}-\pi \\
& \quad+\frac{8(i-1) x_{2}}{2 x_{1}-\pi(i+1) x_{2}+\sqrt{\left[2 x_{1}-\pi(i+1) x_{2}\right]^{2}-8(i-1)^{2} x_{2}^{2}}} \\
& \quad=0,
\end{aligned}
$$

$$
\begin{gathered}
6 \times 10^{4} \times C_{8} \leq \pi n_{1} x_{2} \leq 6 \times 10^{4} \times C_{9}, \\
n_{1} x_{2}-19.1 C_{10} x_{1} \leq 0 \\
C_{14} \leq x_{1} \leq C_{15} \\
C_{12} C_{11} \leq x_{2} \leq C_{13} C_{11} \\
\frac{\pi C_{16}}{180} \leq x_{3} \leq \frac{\pi C_{17}}{180} \\
\mathbf{x} \geq 0
\end{gathered}
$$

where $W(\mathbf{x})=(T(\mathbf{x}))^{-1 / m}, T(\mathbf{x})$ denotes the fatigue life $(h)$ of $\mathrm{V}$-belt for a given decision variable $\mathbf{x}$,

$$
\begin{aligned}
& C_{1}=\left(\frac{1}{7200}\right)^{-1 / m}\left(\frac{\pi}{60000}\right)^{-(m-1) / m}, \quad C_{2}=\frac{1000}{A}, \\
& C_{3}=\frac{\pi y_{0}}{30000 K_{i}}, \quad C_{4}=\frac{\rho_{l}}{A}\left(\frac{\pi}{60000}\right)^{3}, \quad C_{5}=\mu_{v},
\end{aligned}
$$

$m, A, y_{0}, K_{i}, \rho_{l}, \mu_{v}, C, E_{b}$, and $C_{6} \sim C_{17}$ are constants of the model whose meaning are the same as those in [19].

In the real engineering consideration, however, there are many polymorphic uncertain parameters in the optimal design model of maximizing the fatigue life of V-belt, just as described in the section of introduction. Suppose that the experimental constant $C$, the bending modulus $E_{b}$, of elasticity, the carrying power $P$, of a single V-belt, and the rotational speed $n_{1}$, of small pulley, are treated as random parameters with normal distributions, and denoted by $\mathbf{C}, \mathbf{E}_{\mathbf{b}}$, $\mathbf{P}$, and $\mathbf{n}_{1}$, respectively. The transmission rate $i$ is regarded as an interval parameter denoted by $i^{ \pm}=\left[i^{-}, i^{+}\right]$. The parameters $C_{6}, C_{7}, C_{8}, C_{9}, C_{10}, C_{12}, C_{13}, C_{14}, C_{15}$, and $C_{16}$ in the constraints which are associated with the limitations on the bound and performance are considered as fuzzy subsets, denoted by $\widetilde{C_{6}}, \widetilde{C_{7}}, \widetilde{C_{8}}, \widetilde{C_{9}}, \widetilde{C_{10}}, \widetilde{C_{12}}, \widetilde{C_{13}}, \widetilde{C_{14}}, \widetilde{C_{15}}$, and $\widetilde{C_{16}}$, respectively. Furthermore, it is assumed that these parameters are quantified by the following two types of membership functions. For $\widetilde{C_{i}}, i=6,8,12,14,16$, the respective membership function is specified as

$$
\mu_{\widetilde{C_{i}}}(t)= \begin{cases}1-\frac{C_{i}^{u}-t}{\alpha_{c_{i}}}, & C_{i}^{u} \geq t \geq C_{i}^{u}-\alpha_{c_{i}} \\ 0, & \text { otherwise. }\end{cases}
$$

For $\widetilde{C_{i}}, i=7,9,10,13,15$, the corresponding membership functions are, respectively, defined as:

$$
\mu_{\widetilde{C_{i}}}(t)= \begin{cases}1-\frac{t-C_{i}^{l}}{\alpha_{c_{i}}}, & C_{i}^{l} \leq t \leq C_{i}^{l}+\alpha_{c_{i}}, \\ 0, & \text { otherwise. }\end{cases}
$$

The following concept is useful to construct a PUNP model for the problem of maximizing the fatigue life of $\mathrm{V}$ belt.

Definition 1 (multiplication of interval number and fuzzy subset). Let $a^{ \pm}$be an interval number. Let $\widetilde{b}$ be a fuzzy subset with membership function $\mu_{\tilde{b}} \cdot \widetilde{c}^{ \pm}=a^{ \pm} \widetilde{b}$ is said to be the multiplication of $a^{ \pm}$and $\widetilde{b}$ if $\widetilde{c}^{ \pm}$is specified by a fuzzy interval $\left[\widetilde{c}^{-}, \widetilde{c}^{+}\right]$, where $\widetilde{c}^{-}$and $\widetilde{c}^{+}$are two fuzzy subsets quantified, respectively, by the membership functions of $\mu_{a^{-} \widetilde{b}}$ and $\mu_{a^{+} \widetilde{b}}$ where $\mu_{\tilde{b}}(x)=\mu_{a^{-} \widetilde{b}}\left(a^{-} x\right)=\mu_{a^{+} \tilde{b}}\left(a^{+} x\right)$.

From the above definition, it is easily seen that $a^{ \pm} \widetilde{b}$ is a special fuzzy process $t \tilde{b}$, where $t \in\left[a^{-}, a^{+}\right]$. From the theory of fuzzy mathematics, we know that this fuzzy process is determined by the two boundary values: $a^{-} \widetilde{b}$ and $a^{+} \widetilde{b}$.

With the preparation above, the problem of maximizing the V-belt's fatigue life is formulated into the following nonlinear PUNP model:

$$
\begin{aligned}
& \min W(\mathbf{x})=C_{1} \mathbf{C}^{-1 / m} \mathbf{n}_{1}^{-(m-1) / m} x_{1}^{-1 / m} x_{2}^{-(m-1) / m} \\
& \times\left[C_{2} \mathbf{P}\left(1-e^{-C_{5} x_{3}}\right)^{-1}+C_{3} \mathbf{n}_{1} \mathbf{E}_{\mathbf{b}}+C_{4} \mathbf{n}_{1}^{3} x_{2}^{3}\right] \\
& \text { s.t. } \quad{\widetilde{C_{6}}}^{ \pm} x_{2}-\left(2 x_{1}-\pi I_{1}^{ \pm} x_{2}+\sqrt{\left[2 x_{1}-\pi I_{1}^{ \pm} x_{2}\right]^{2}-8 I^{ \pm} x_{2}^{2}}\right) \\
& \leq 0 \text {, } \\
& \left(2 x_{1}-\pi I_{1}^{ \pm} x_{2}+\sqrt{\left[2 x_{1}-\pi I_{1}^{ \pm} x_{2}\right]^{2}-8 I^{ \pm} x_{2}^{2}}\right)-{\widetilde{C_{7}}}^{ \pm} x_{2} \\
& \leq 0 \text {, } \\
& \left(x_{3}-\pi\right)\left(2 x_{1}-\pi I_{1}^{ \pm} x_{2}+\sqrt{\left[2 x_{1}-\pi I_{1}^{ \pm} x_{2}\right]^{2}-8 I^{ \pm} x_{2}^{2}}\right) \\
& +8 I_{2}^{ \pm} x_{2}=0 \\
& 6 \times 10^{4} \times \widetilde{C_{8}} \leq \pi \mathbf{n}_{1} x_{2} \leq 6 \times 10^{4} \times \widetilde{C_{9}}, \\
& \mathbf{n}_{1} x_{2}-19.1 \widetilde{C_{10}} x_{1} \leq 0, \\
& \widetilde{C_{14}} \leq x_{1} \leq \widetilde{C_{15}} \\
& \widetilde{C_{12}} \widetilde{C_{11}} \leq x_{2} \leq \widetilde{C_{13}} \widetilde{C_{11}} \text {, } \\
& \frac{\pi \widetilde{C_{16}}}{180} \leq x_{3} \leq \frac{\pi C_{17}}{180}, \\
& \mathbf{x} \geq 0 \text {, }
\end{aligned}
$$


where ${\widetilde{C_{6}}}^{ \pm}$and $\widetilde{C_{7}}{ }^{ \pm}$are two positive fuzzy interval-parameters (see Definition 1) denoted by

$$
\begin{gathered}
{\widetilde{C_{6}}}^{ \pm}=8 \widetilde{C_{6}} I_{1}^{ \pm}, \quad{\widetilde{C_{7}}}^{ \pm}=8 \widetilde{C_{7}} I_{1}^{ \pm}, \quad I^{ \pm}=\left(I_{2}^{ \pm}\right)^{2}, \\
I_{1}^{ \pm}=i^{ \pm}+1, \quad I_{2}^{ \pm}=i^{ \pm}-1 .
\end{gathered}
$$

\section{Equivalent Formulation of PUNP Model}

In this section, the PUNP model (5) for the problem of maximizing the fatigue life of $\mathrm{V}$-belt will be be transformed into an equivalent formulation where only interval parameters are involved.

We first present the following two definitions.

Definition 2. Let $\widetilde{A}$ be a fuzzy subset on a nonempty set $X$, and let $a_{0}$ be a given real number. An inequality $\widetilde{A} \geq a_{0}$ is said to hold if for any $\beta \in(0,1], \widetilde{A}_{\beta} \geq a_{0}$ holds.

Remark 3. By Definition 2, we can approximate a fuzzy inequality by an interval inequality by fixing a suitable degree of membership. Certainly, if $\widetilde{A}$ is replaced by its fuzzy expectation, then the fuzzy inequality is transformed into an ordinary inequality. However, in this paper, we treat a fuzzy inequality in a more flexible manner, just as in Definition 2.

Definition 4. Let $\xi$ be a random variable, and let $\xi_{0}$ be a given real number. An inequality $\xi x \leq \xi_{0}$ is said to hold if for any $\alpha \in(0,1), P\left(\xi_{x} \leq \xi_{0}\right) \geq \alpha$, where the operator $P(\cdot)$ denotes the probability of random occurrence.

Remark 5. By Definition 4, a random inequality may be approximated by an ordinary inequality for a given chancelevel $\alpha$.

Next, we turn to present some theorems that will be employed to deal with the polymorphic uncertainty.

The following result is useful to transform an inequality involved with random variables and fuzzy subsets into an interval one.

Theorem 6. Suppose that $\xi$ is a random variable with normal distribution, and $\widetilde{A}$ is a fuzzy subset on a nonempty set $X$. Then the following inequalities:

$$
\xi x \leq \widetilde{A}, \quad x>0,
$$

hold if and only if for any $\alpha \in(0,1)$, the inequality

$$
\left[E(\xi)+D(\xi) \Phi^{-1}(\alpha)\right] \cdot x \leq \widetilde{A}_{\beta}
$$

holds for $\beta \in(0,1]$, where $\Phi^{-1}:[0,1] \rightarrow R$ is the inverse function of the cumulative distribution function of a random variable with standard normal distribution, and $D(\xi)$ denotes the standard deviation of the random variable $\xi$.

Proof. Firstly, (7) can be rewritten as

$$
\xi \leq \frac{\widetilde{A}}{x}, \quad x>0 .
$$

By Definition 4, we know that for any $\alpha \in(0,1)$,

$$
P\left(\xi \leq \frac{\widetilde{A}}{x}\right) \geq \alpha .
$$

Since $\xi$ is a random variable with normal distribution, we have

$$
\begin{aligned}
P\left(\xi \leq \frac{\widetilde{A}}{x}\right) & =P\left(\zeta \leq \frac{(\widetilde{A} / x)-E(\xi)}{D(\xi)}\right) \\
& =\Phi\left(\frac{(\widetilde{A} / x)-E(\xi)}{D(\xi)}\right) \geq \alpha,
\end{aligned}
$$

where $\zeta$ is the random variable with standard normal distribution, $\Phi: R \rightarrow[0,1]$ is the cumulative distribution function of $\zeta$. From the increasing monotonicity of $\Phi$, it follows that

$$
\frac{(\widetilde{A} / x)-E(\xi)}{D(\xi)} \geq \Phi^{-1}(\alpha)
$$

It yields

$$
\widetilde{A} \geq\left[E(\xi)+D(\xi) \Phi^{-1}(\alpha)\right] \cdot x .
$$

By Definition 2, the inequality (13) says that for any $\beta \in(0,1]$,

$$
\widetilde{A}_{\beta} \geq\left[E(\xi)+D(\xi) \Phi^{-1}(\alpha)\right] \cdot x
$$

holds. The desired result is proved.

Similarly, we can prove the following theorem.

Theorem 7. Suppose that $\xi$ is a random variable with normal distribution, and $\widetilde{A}$ is a fuzzy subset on a nonempty set $X$. Then,

$$
\xi x \geq \widetilde{A}, \quad x>0
$$

if and only if for any $\alpha \in(0,1)$, the following inequality:

$$
\left[E(\xi)+D(\xi) \Phi^{-1}(1-\alpha)\right] \cdot x \geq \widetilde{A}_{\beta}
$$

holds for $\beta \in(0,1]$.

In particular, if $\widetilde{A}$ is a fuzzy subset with the membership function $\mu_{\widetilde{A}}$, given by

$$
\mu_{\widetilde{A}}(x)= \begin{cases}\frac{x-a}{b-a}, & x \in[a, b] \\ 1, & x \in[b, c] \\ \frac{d-x}{d-c}, & x \in[c, d] \\ 0, & \text { otherwise }\end{cases}
$$

then for any fixed $\beta \in(0,1], \widetilde{A}_{\beta}=[a+\beta(b-a), d-\beta(d-$ $c)$ ]. Suppose that $\xi \sim N\left(\mu_{0}, \sigma_{0}{ }^{2}\right)$. Then for the given $\beta$ and $\alpha \in(0,1)$, the inequality $\xi x \leq \widetilde{A}$ can be approximated by an interval inequality:

$$
\left[\mu_{0}+\sigma_{0} \Phi^{-1}(\alpha)\right] x \leq[a+\beta(b-a), d-\beta(d-c)] .
$$


Generally, Theorems 6 and 7 can be used to obtain a deterministic interval equivalent formulation for approximating the forth and the fifth constraints in model (5).

The following lemma is useful to treat the random parameters in the objective function.

Lemma 8. Assume that $\mathbf{C}, \mathbf{n}_{1}, \mathbf{E}_{\mathbf{b}}$, and $\mathbf{P}$ are mutually independent random parameters. Then,

$$
\begin{aligned}
E(W(\mathbf{x}))= & C_{1} E\left(\mathbf{C}^{-1 / m}\right) E\left(\mathbf{n}_{\mathbf{1}}^{-(m-1) / m}\right) x_{1}^{-1 / m} x_{2}^{-(m-1) / m} \\
\times & C_{2} E(\mathbf{P})\left(1-e^{-C_{5} x_{3}}\right)^{-1}+C_{3} E\left(\mathbf{n}_{\mathbf{1}}\right) E\left(\mathbf{E}_{\mathbf{b}}\right) \\
& \left.+C_{4} E\left(\mathbf{n}_{\mathbf{1}}^{3}\right) x_{2}^{3}\right],
\end{aligned}
$$

where $E(\mathbf{X})$ denotes the expectation of a random variable $\mathbf{X}$.

Proof. The result is directly from the mutual independence of the random parameters.

The following useful lemma is directly from the famous Nguyen's theorem in [21].

Lemma 9. Let $f: R^{2} \rightarrow R$ be given by $f(x, y)=x y$. Suppose that $\widetilde{A}_{\alpha}=\left[a_{1}(\alpha), a_{2}(\alpha)\right]$ and $\widetilde{B}_{\alpha}=\left[b_{1}(\alpha), b_{2}(\alpha)\right]$. Then,

$$
\begin{gathered}
{[f(\widetilde{A}, \widetilde{B})]_{\alpha}=[\widetilde{A} \widetilde{B}]_{\alpha}} \\
=\left[\operatorname { m i n } \left\{a_{1}(\alpha) b_{1}(\alpha), a_{1}(\alpha) b_{2}(\alpha), a_{2}(\alpha) b_{1}(\alpha),\right.\right. \\
\left.\quad a_{2}(\alpha) b_{2}(\alpha)\right\}, \\
\max \left\{a_{1}(\alpha) b_{1}(\alpha), a_{1}(\alpha) b_{2}(\alpha), a_{2}(\alpha) b_{1}(\alpha),\right. \\
\left.\left.\quad a_{2}(\alpha) b_{2}(\alpha)\right\}\right] .
\end{gathered}
$$

In particular, if $\widetilde{A}$ and $\widetilde{B}$ are two positive fuzzy numbers, then

$$
[\widetilde{A} \widetilde{B}]_{\alpha}=\left[a_{1}(\alpha) b_{1}(\alpha), a_{2}(\alpha) b_{2}(\alpha)\right] .
$$

Lemma 9 is useful to handle the seventh constraint in model (5).

From Theorems 6 and 7, Lemmas 8 and 9, it is concluded that for any given satisfactory level $\alpha_{1}$ which is used to calculate an $\alpha_{1}$-cut for a fuzzy number and a confidence level $\alpha_{2}$ (hereinafter, we take $\alpha_{1}=\alpha_{2}=\alpha$ ), model
(5) is transformed into the following interval programming problem:

$$
\begin{aligned}
\min E(W(\mathbf{x}))=C_{1} E\left(\mathbf{C}^{-1 / m}\right) & E\left(\mathbf{n}_{\mathbf{1}}^{-(m-1) / m}\right) x_{1}^{-1 / m} \\
\times x_{2}^{-(m-1) / m}[ & C_{2} E(\mathbf{P})\left(1-e^{-C_{5} x_{3}}\right)^{-1} \\
& +C_{3} E\left(\mathbf{n}_{\mathbf{1}}\right) E\left(\mathbf{E}_{\mathbf{b}}\right) \\
& \left.+C_{4} E\left(\mathbf{n}_{\mathbf{1}}^{3}\right) x_{2}^{3}\right]
\end{aligned}
$$

s.t. $\left[{\widetilde{C_{6}}}^{ \pm}\right]_{\alpha} x_{2}-\left(2 x_{1}-\pi I_{1}^{ \pm} x_{2}\right.$

$$
\left.+\sqrt{\left[2 x_{1}-\pi I_{1}^{ \pm} x_{2}\right]^{2}-8\left(I_{2}^{ \pm}\right)^{2} x_{2}^{2}}\right)
$$

$\leq 0$,

$\left(2 x_{1}-\pi I_{1}^{ \pm} x_{2}+\sqrt{\left[2 x_{1}-\pi I_{1}^{ \pm} x_{2}\right]^{2}-8\left(I_{2}^{ \pm}\right)^{2} x_{2}^{2}}\right)-\left[{\widetilde{C_{7}}}^{ \pm}\right]_{\alpha} x_{2}$ $\leq 0$,

$$
\begin{aligned}
& \left(x_{3}-\pi\right)\left(2 x_{1}-\pi I_{1}^{ \pm} x_{2}+\sqrt{\left[2 x_{1}-\pi I_{1}^{ \pm} x_{2}\right]^{2}-8 I^{ \pm} x_{2}^{2}}\right) \\
& \quad+8 I_{2}^{ \pm} x_{2}=0 \\
& {\left[\pi E\left(\mathbf{n}_{1}\right)+\pi \Phi^{-1}(1-\alpha) D\left(\mathbf{n}_{1}\right)\right] \cdot x_{2} \geq 6 \times 10^{4} \times\left[\widetilde{C_{8}}\right]_{\alpha},} \\
& {\left[\pi E\left(\mathbf{n}_{1}\right)+\pi \Phi^{-1}(\alpha) D\left(\mathbf{n}_{1}\right)\right] \cdot x_{2} \leq 6 \times 10^{4} \times\left[\widetilde{C_{9}}\right]_{\alpha},} \\
& {\left[E\left(\mathbf{n}_{\mathbf{1}}\right)+\Phi^{-1}(\alpha) D\left(\mathbf{n}_{1}\right)\right] x_{2}-19.1\left[\widetilde{C_{10}}\right]_{\alpha} x_{1} \leq 0,} \\
& {\left[\widetilde{C_{14}}\right]_{\alpha} \leq x_{1} \leq\left[\widetilde{C_{15}}\right]_{\alpha},} \\
& {\left[\widetilde{C_{12}} \widetilde{C_{11}}\right]_{\alpha} \leq x_{2} \leq\left[\widetilde{C_{11}} \widetilde{C_{13}}\right]_{\alpha},} \\
& \frac{\pi}{180}\left[\widetilde{C_{16}}\right]_{\alpha} \leq x_{3} \leq \frac{\pi}{180} C_{17}, \\
& \mathbf{x} \geq 0 \\
& \alpha \in[0,1]
\end{aligned}
$$

where $\left[{\widetilde{C_{6}}}^{ \pm}\right]_{\alpha}=\left[\left(\left[{\widetilde{C_{6}}}^{-}\right]_{\alpha}\right)^{-},\left(\left[{\widetilde{C_{6}}}^{+}\right]_{\alpha}\right)^{+}\right],\left[{\widetilde{C_{7}}}^{ \pm}\right]_{\alpha}=\left[\left(\left[{\widetilde{C_{7}}}^{-}\right]_{\alpha}\right)^{-}\right.$, $\left.\left(\left[\widetilde{C}_{7}^{+}\right]_{\alpha}\right)^{+}\right]$.

Remark 10. In model (22), the given satisfactory level $\alpha$ is used to determine an $\alpha$-cut for a fuzzy number. For a fuzzy number $\widetilde{A}$ with a concave membership function, its $\alpha$-cut $\widetilde{A}_{\alpha}$ is an interval, denoted by $\widetilde{A}_{\alpha}^{ \pm}$. By decomposition theorem, we know

$$
\widetilde{A}=\bigcup_{\alpha \in[0,1]} \alpha \widetilde{A}_{\alpha}^{ \pm} .
$$

Hence, $\widetilde{A}_{\alpha}$ is an approximate description of $\widetilde{A}$.

Similarly, the confidence level $\alpha$ is used to determine an interval-value to approximate a random number. 
Remark 11. In the practical engineering applications, the values of the satisfactory level and the confidence level should be determined by the known information with regard to the uncertain parameters. The satisfactory level is an index for describing fuzzy parameter. It can be determined by the theory of fuzzy set. For example, the value of satisfactory level can be inferred by the two-level fuzzy synthetic evaluation method (see [22]) according to the subjective information in regard to the fuzzy parameter such as the design level, the manufacturing level, and the importance of structure, while the confidence level is an index for describing random parameter and its value can be obtained according to the corresponding objective information. For instance, we can get the expectation and the probability distribution of a random parameter by a mass of experiments which are helpful to infer the confidence level. In this paper, in order to simplify the calculation, we assume that all the values of the satisfactory level and the confidence level are equal.

\section{Two-Step Based Sampling Algorithm}

In this section, a two-step based sampling algorithm is developed to find an interval optimal solution for the original problem.

Firstly, model (22) is decomposed into two standard nonlinear programming subproblems to find an interval optimal solution, which is based on the concepts of maximal and minimal range inequalities for describing an interval inequality similar to those presented in [20].

To begin, the following subproblem is solved to obtain the lower bound of the optimal objective value of model (22) by sampling method:

$$
\begin{aligned}
& \min E(W(\mathbf{x}))=C_{1} E\left(\mathbf{C}^{-1 / m}\right) E\left(\mathbf{n}_{\mathbf{1}}^{-(m-1) / m}\right) \\
& \times x_{1}^{-1 / m} x_{2}^{-(m-1) / m} \\
& \times\left[C_{2} E(\mathbf{P})\left(1-e^{-C_{5} x_{3}}\right)^{-1}\right. \\
& \left.+C_{3} E\left(\mathbf{n}_{\mathbf{1}}\right) E\left(\mathbf{E}_{\mathbf{b}}\right)+C_{4} E\left(\mathbf{n}_{\mathbf{1}}^{3}\right) x_{2}^{3}\right] \\
& \text { s.t. } 2 x_{1}-\pi I_{1}^{-} x_{2}+\sqrt{\left(2 x_{1}-\pi I_{1}^{-} x_{2}\right)^{2}-8\left(I_{2}^{-}\right)^{2} x_{2}^{2}} \\
& -\left[{\widetilde{C_{6}}}^{-}\right]_{\alpha}^{-} x_{2} \geq 0 \text {, } \\
& -\left(2 x_{1}-\pi I_{1}^{+} x_{2}+\sqrt{\left(2 x_{1}-\pi I_{1}^{+} x_{2}\right)^{2}-8\left(I_{2}^{+}\right)^{2} x_{2}^{2}}\right) \\
& +\left[\widetilde{C}_{7}^{+}\right]_{\alpha}^{+} x_{2} \geq 0 \\
& -\left(x_{3}-\pi\right)\left(2 x_{1}-\pi I_{1}^{-} x_{2}+\sqrt{\left[2 x_{1}-\pi I_{1}^{-} x_{2}\right]^{2}-8 I^{-} x_{2}^{2}}\right) \\
& -8 I_{2}^{-} x_{2} \geq 0 \\
& \left(x_{3}-\pi\right)\left(2 x_{1}-\pi I_{1}^{+} x_{2}+\sqrt{\left[2 x_{1}-\pi I_{1}^{+} x_{2}\right]^{2}-8 I^{+} x_{2}^{2}}\right)
\end{aligned}
$$

$$
\begin{gathered}
+8 I_{2}^{+} x_{2} \geq 0 \\
{\left[\pi E\left(\mathbf{n}_{\mathbf{1}}\right)+\pi \Phi^{-1}(1-\alpha) D\left(\mathbf{n}_{\mathbf{1}}\right)\right] \cdot x_{2} \geq 6 \times 10^{4} \times\left[\widetilde{C_{8}}\right]_{\alpha}^{-}} \\
{\left[\pi E\left(\mathbf{n}_{\mathbf{1}}\right)+\pi \Phi^{-1}(\alpha) D\left(\mathbf{n}_{\mathbf{1}}\right)\right] \cdot x_{2} \leq 6 \times 10^{4} \times\left[\widetilde{C_{9}}\right]_{\alpha}^{+}} \\
-\left[E\left(\mathbf{n}_{1}\right)+\Phi^{-1}(\alpha) D\left(\mathbf{n}_{1}\right)\right] x_{2}+19.1\left[\widetilde{C_{10}}\right]_{\alpha}^{+} x_{1} \geq 0 \\
\left(\left[\widetilde{C_{15}}\right]_{\alpha}\right)^{+} \geq x_{1} \geq\left(\left[\widetilde{C_{14}}\right]_{\alpha}\right)^{-} \\
\left(\left[\widetilde{C_{11}} \widetilde{C_{13}}\right]_{\alpha}\right)^{+} \geq x_{2} \geq\left(\left[\widetilde{C_{11}} \widetilde{C_{12}}\right]_{\alpha}\right)^{-} \\
\frac{\pi}{180} C_{17} \geq x_{3} \geq\left(\frac{\pi}{180}\left[\widetilde{C_{16}}\right]_{\alpha}\right)^{-} \\
\mathbf{x} \geq 0 .
\end{gathered}
$$

The obtained solution is represented as $\mathbf{x}_{*}^{a}=\left(x_{1 *}^{a}, x_{2 *}^{a}\right.$, $\left.x_{3 *}^{a}\right)$ and denotes the corresponding value of the objective function by $W_{*}^{-}$.

Next, the second subproblem is solved to obtain the upper bound of the optimal objective value of model (22) by sampling method:

$\min$

$$
\begin{aligned}
& E(W(\mathbf{x})) \\
& =C_{1} E\left(\mathbf{C}^{-1 / m}\right) E\left(\mathbf{n}_{\mathbf{1}}^{-(m-1) / m}\right) x_{1}^{-1 / m} x_{2}^{-(m-1) / m} \\
& \quad \times\left[C_{2} E(\mathbf{P})\left(1-e^{-C_{5} x_{3}}\right)^{-1}+C_{3} E\left(\mathbf{n}_{1}\right) E\left(\mathbf{E}_{\mathbf{b}}\right)\right. \\
& \left.\quad+C_{4} E\left(\mathbf{n}_{1}^{3}\right) x_{2}^{3}\right]
\end{aligned}
$$

s.t. $\quad 2 x_{1}-\pi I_{1}^{+} x_{2}+\sqrt{\left(2 x_{1}-\pi I_{1}^{+} x_{2}\right)^{2}-8\left(I_{2}^{+}\right)^{2} x_{2}^{2}}$

$$
-\left[{\widetilde{C_{6}}}^{+}\right]_{\alpha}^{+} x_{2}
$$

$\geq 0$,

$$
\begin{aligned}
& -\left(2 x_{1}-\pi I_{1}^{-} x_{2}+\sqrt{\left(2 x_{1}-\pi I_{1}^{-} x_{2}\right)^{2}-8\left(I_{2}^{-}\right)^{2} x_{2}^{2}}\right) \\
& +\left[{\widetilde{C_{7}}}^{-}\right]_{\alpha}^{-} x_{2}
\end{aligned}
$$

$\geq 0$,

$$
\begin{aligned}
&-\left(x_{3}-\pi\right)\left(2 x_{1}-\pi I_{1}^{-} x_{2}+\sqrt{\left[2 x_{1}-\pi I_{1}^{-} x_{2}\right]^{2}-8 I^{-} x_{2}^{2}}\right) \\
&-8 I_{2}^{-} x_{2} \\
& \geq 0 \\
&\left(x_{3}-\pi\right)\left(2 x_{1}-\pi I_{1}^{+} x_{2}+\sqrt{\left[2 x_{1}-\pi I_{1}^{+} x_{2}\right]^{2}-8 I^{+} x_{2}^{2}}\right) \\
& \quad+8 I_{2}^{+} x_{2} \\
& \geq 0
\end{aligned}
$$




$$
\begin{aligned}
& {\left[\pi E\left(\mathbf{n}_{1}\right)+\pi \Phi^{-1}(1-\alpha) D\left(\mathbf{n}_{1}\right)\right] \cdot x_{2} \geq 6 \times 10^{4} \times\left[\widetilde{C_{8}}\right]_{\alpha}^{+}} \\
& {\left[\pi E\left(\mathbf{n}_{1}\right)+\pi \Phi^{-1}(\alpha) D\left(\mathbf{n}_{1}\right)\right] \cdot x_{2} \leq 6 \times 10^{4} \times\left[\widetilde{C_{9}}\right]_{\alpha}^{-}} \\
& -\left[E\left(\mathbf{n}_{1}\right)+\Phi^{-1}(\alpha) D\left(\mathbf{n}_{1}\right)\right] x_{2}+19.1\left[\widetilde{C_{10}}\right]_{\alpha}^{-} x_{1} \geq 0 \\
& \left(\left[\widetilde{C_{15}}\right]_{\alpha}\right)^{-} \geq x_{1} \geq\left(\left[\widetilde{C_{14}}\right]_{\alpha}\right)^{+} \\
& \left(\left[\widetilde{C_{11}} \widetilde{C_{13}}\right]_{\alpha}\right)^{-} \geq x_{2} \geq\left(\left[\widetilde{C_{11}} \widetilde{C_{12}}\right]_{\alpha}\right)^{+} \\
& \frac{\pi}{180} C_{17} \geq x_{3} \geq\left(\frac{\pi}{180}\left[\widetilde{C_{16}}\right]_{\alpha}\right)^{+} \\
& \mathbf{x} \geq 0 .
\end{aligned}
$$

The obtained solution is represented as $\mathbf{x}_{*}^{b}=\left(x_{1 *}^{b}, x_{2 *}^{b}\right.$, $\left.x_{3 *}^{b}\right)$ and denotes the corresponding value of the objective function by $W_{*}^{+}$.

Then, for a given satisfaction level $\alpha$, an interval optimal solution, denoted as $\mathbf{x}_{*}^{ \pm}$, is specified as the following interval vector:

$$
\begin{gathered}
\left(\left[\min \left\{x_{1 *}^{a}, x_{1 *}^{b}\right\}, \max \left\{x_{1 *}^{a}, x_{1 *}^{b}\right\}\right],\right. \\
{\left[\min \left\{x_{2 *}^{a}, x_{2 *}^{b}\right\}, \max \left\{x_{2 *}^{a}, x_{2 *}^{b}\right\}\right],} \\
\left.\left[\min \left\{x_{3 *}^{a}, x_{3 *}^{b}\right\} \max \left\{x_{3 *}^{a}, x_{3 *}^{b}\right\}\right]\right)^{T} .
\end{gathered}
$$

The corresponding interval optimal value of the objective function is $W_{*}^{ \pm}=\left[W_{*}^{-}, W_{*}^{+}\right]$.

The procedure of a two-step based sampling algorithm is detailed as follows.

Algorithm 12 (Two-step based sampling algorithm).

Step 0 . Choose a capacity $N_{k}$ of sampling. Set $k:=0$.

Step 1. According to the given distribution functions of random parameters $\mathbf{C}, \mathbf{E}_{\mathbf{b}}, \mathbf{P}$, and $\mathbf{n}_{\mathbf{1}}$, independently and randomly distributed samples are generated. Let their realizations be referred to as $\widehat{\mathbf{C}}, \widehat{\mathbf{E}}_{\mathbf{b}}, \widehat{\mathbf{P}}$, and $\widehat{\mathbf{n}}_{\mathbf{1}}$, respectively. It is clear that all of them are $N_{k}$-dimensional vectors.

Step 2. Use an optimization solver to obtain the solution of the following problem:

$$
\begin{aligned}
\min E(W(\mathbf{x}))= & C_{1} \frac{1}{N_{k}^{2}} \sum_{l=1}^{N_{k}}(\widehat{\mathbf{C}})_{l}^{-1 / m} \\
& \times \sum_{l=1}^{N_{k}}\left(\widehat{\mathbf{n}}_{\mathbf{1}}\right)_{l}^{-(m-1) / m} x_{1}^{-1 / m} x_{2}^{-(m-1) / m}
\end{aligned}
$$

$$
\begin{gathered}
\times\left[C_{2} \frac{1}{N_{k}} \sum_{l=1}^{N_{k}}(\widehat{\mathbf{P}})_{l}\left(1-e^{-C_{5} x_{3}}\right)^{-1}\right. \\
+C_{3} \frac{1}{N_{k}^{2}} \sum_{l=1}^{N_{k}}\left(\widehat{\mathbf{n}}_{\mathbf{1}}\right)_{l} \sum_{l=1}^{N_{k}}\left(\widehat{\mathbf{E}}_{\mathbf{b}}\right)_{l} \\
\left.+C_{4} \frac{1}{N_{k}} \sum_{l=1}^{N_{k}}\left(\widehat{\mathbf{n}}_{\mathbf{1}}\right)_{l}^{3} x_{2}^{3}\right]
\end{gathered}
$$

s.t. $\mathbf{x} \in \mathscr{F}_{1}$,

where $\mathscr{F}_{1}$ denotes the feasibility region of model (4.1). Denote $(\widehat{\mathbf{C}})_{l},\left(\widehat{\mathbf{n}}_{1}\right)_{l},\left(\widehat{\mathbf{E}}_{\mathbf{b}}\right)_{l}$, and $(\widehat{\mathbf{P}})_{l}$ the the $l$ th component of the vectors $\widehat{\mathbf{C}}, \widehat{\mathbf{n}}_{1}, \widehat{\mathbf{E}}_{\mathbf{b}}$, and $\widehat{\mathbf{P}}$, respectively. Let the solution be denoted as $\mathbf{x}_{*}^{a}\left(N_{k}\right)$, and the corresponding value of the objective function be referred to as $W_{*}^{-}\left(N_{k}\right)$, that is, we have the value of the objective function $T_{*}^{+}\left(N_{k}\right)$ correspondingly.

Step 3. Use an optimization solver to obtain the solution of the following problem:

$$
\begin{aligned}
\min E(W(\mathbf{x}))= & C_{1} \frac{1}{N_{k}^{2}} \sum_{l=1}^{N_{k}}(\widehat{\mathbf{C}})_{l}^{-1 / m} \\
& \times \sum_{l=1}^{N_{k}}\left(\widehat{\mathbf{n}}_{1}\right)_{l}^{-(m-1) / m} x_{1}^{-1 / m} x_{2}^{-(m-1) / m} \\
& \times\left[C_{2} \frac{1}{N_{k}} \sum_{l=1}^{N_{k}}(\widehat{\mathbf{P}})_{l}\left(1-e^{-C_{5} x_{3}}\right)^{-1}\right. \\
& +C_{3} \frac{1}{N_{k}^{2}} \sum_{l=1}^{N_{k}}\left(\widehat{\mathbf{n}}_{1}\right)_{l} \sum_{l=1}^{N_{k}}\left(\widehat{\mathbf{E}}_{\mathbf{b}}\right)_{l} \\
& \left.+C_{4} \frac{1}{N_{k}} \sum_{l=1}^{N_{k}}\left(\widehat{\mathbf{n}}_{1}\right)_{l}^{3} x_{2}^{3}\right]
\end{aligned}
$$

s.t. $\quad \mathbf{x} \in \mathscr{F}_{2}$,

where $\mathscr{F}_{2}$ denotes the feasibility region of model (4.2). Let the solution be denoted as $\mathbf{x}_{*}^{b}\left(N_{k}\right)$, and the corresponding value of the objective function be referred to as $W_{*}^{+}\left(N_{k}\right)$, that is, we have the value of the objective function $T_{*}^{-}\left(N_{k}\right)$ correspondingly.

Step 4. If the termination criterion is satisfied, then output the interval optimal solution $\mathbf{x}_{*}^{ \pm}\left(N_{k}\right)=\left[\min \left\{\mathbf{x}_{*}^{a}\left(N_{k}\right)\right.\right.$, $\left.\left.\mathbf{x}_{*}^{b}\left(N_{k}\right)\right\}, \max \left\{\mathbf{x}_{*}^{a}\left(N_{k}\right), \mathbf{x}_{*}^{b}\left(N_{k}\right)\right\}\right]$ and the corresponding interval optimal value $W_{*}^{ \pm}\left(N_{k}\right)$ as well as $T_{*}^{ \pm}\left(N_{k}\right)$, and the algorithm stops. Otherwise, set $k:=k+1$, and choose $N_{k} \geq N_{k-1}$. Return to Step 1 .

Remark 13. The proposed optimization method in this paper is not a general method to solve a generic uncertain nonlinear programming problem, but it is suitable for a type of uncertain models just as (5) from the optimal design of V-belt drive. In particular, the model has the following properties. 
TABLE 1: Interval optimal solutions with different choices of satisfaction level $\alpha$.

\begin{tabular}{|c|c|c|c|c|c|c|}
\hline$\alpha$ & $\mathbf{x}_{*}^{a}$ & $\mathbf{x}_{*}^{b}$ & $W_{*}^{-}$ & $W_{*}^{+}$ & $T_{*}^{-}$ & $T_{*}^{+}$ \\
\hline 0.50 & $(4366.877,161.000,2.874)$ & $(4000.847,154.000,2.858)$ & 0.351 & 0.367 & 61547 & 101366 \\
\hline 0.60 & $(4300.629,159.600,2.872)$ & $(4000.847,154.000,2.858)$ & 0.354 & 0.367 & 61547 & 92186 \\
\hline 0.70 & $(4232.171,158.200,2.869)$ & $(4000.847,154.000,2.858)$ & 0.357 & 0.367 & 61547 & 83676 \\
\hline 0.75 & $(4200.333,157.500,2.868)$ & $(4000.847,154.000,2.858)$ & 0.358 & 0.367 & 61547 & 79741 \\
\hline 0.80 & $(4167.163,156.800,2.867)$ & $(4000.847,154.000,2.858)$ & 0.360 & 0.367 & 61547 & 75935 \\
\hline 0.85 & $(4134.124,156.100,2.865)$ & $(4000.847,154.000,2.858)$ & 0.362 & 0.367 & 61547 & 72290 \\
\hline 0.90 & $(4101.215,155.400,2.864)$ & $(4000.847,154.000,2.858)$ & 0.363 & 0.367 & 61547 & 68801 \\
\hline 0.95 & $(4068.438,154.700,2.862)$ & $(4000.847,154.000,2.858)$ & 0.365 & 0.367 & 61547 & 65461 \\
\hline 0.975 & $(4052.098,154.350,2.862)$ & $(4000.847,154.000,2.858)$ & 0.366 & 0.367 & 61547 & 63846 \\
\hline
\end{tabular}

(1) The constraints is involved with some nonlinear interval inequalities and equalities as shown by Lemmas 8 and 9 in [20].

(2) The expectation of the objective function can be computed.

\section{Case Study}

In this section, the proposed method will be applied to solve a real design problem of maximizing the $V$-belt's fatigue life.

Suppose that the properties of uncertain parameters of the PUNP model (5) in this case study are the same as those in the case study of [19]. For different choices of the satisfaction level $\alpha$, the interval optimal solutions and its corresponding optimal values are obtained by the proposed method as shown in Table 1.

From the numerical results in Table 1, the following is concluded:

(1) With increasing values of the satisfaction level $\alpha$, the upper bound of the interval optimal solution $\mathbf{x}_{*}^{a}$ becomes smaller, and the size of the interval solution becomes smaller too.

(2) With increasing values of the satisfaction level $\alpha$, the lower bound of the interval optimal value $W_{*}^{-}$ becomes larger, and $T_{*}^{+}$becomes smaller correspondingly.

(3) For the given design environment, the lower bound of the interval optimal solution $\mathbf{x}_{*}^{b}$, the upper bound of the interval optimal value $W_{*}^{+}$, and $T_{*}^{-}$is independent of the satisfaction level $\alpha$. At the attained optimal solution, only the second and the third constraints in model (25) are efficient.

\section{Conclusion}

In this paper, we have constructed a nonlinear PUNP model for maximizing the fatigue life in the design of $\mathrm{V}$-belt under polymorphic uncertain environment. For a given suitable satisfaction level and any confidence level, an interval optimal solution for the original problem is found by developing a two-step based sampling algorithm. Case study showed the efficiency of the constructed model and the algorithm in solving such an uncertain mechanical design problem.

\section{Acknowledgments}

The authors would like to express their thanks to the anonymous referees for their suggestive comments that greatly improved the presentation of this paper. This research was supported by the National Natural Science Foundation of China (Grant nos. 71221061, 70921001).

\section{References}

[1] S. J. Zhang, Z. Wan, and G. L. Liu, "Global optimization design method for maximizing the capacity of V-belt drive," Science China Technological Sciences E, vol. 54, no. 1, pp. 140-147, 2011.

[2] C. S. Yang, "Design optimization of belt tramsmission by intelligent algorithm," in Proceedings of the International Conference on Computational Intelligence and Software Engineering (CiSE '09), pp. 1-4, December 2009.

[3] C. J. Shieh and W. H. Chen, "Effect of angular speed on behavior of a V-belt drive system," International Journal of Mechanical Sciences, vol. 44, no. 9, pp. 1879-1892, 2002.

[4] G. Čepon, L. Manin, and M. Boltežar, "Experimental identification of the contact parameters between a V-ribbed belt and a pulley," Mechanism and Machine Theory, vol. 45, no. 10, pp. 1424-1433, 2010.

[5] S. J. Zhang, Z. Wan, and G. L. Liu, "Global optimization design of V-belt fatigue life," China Mechanical Engineering, vol. 22, no. 4, pp. 403-407, 2011.

[6] Z. Wan, F. Z. Meng, A. Y. Hao, and Y. L. Wang, "Fuzzy and stochastic parameters-based prediction method for the components of alkali in the sintering process of aluminium," Fuzzy Systems and Mathematics, vol. 25, no. 3, pp. 163-167, 2011.

[7] X. Chen, C. Zhang, and M. Fukushima, "Robust solution of monotone stochastic linear complementarity problems," Mathematical Programming, vol. 117, no. 1-2, pp. 51-80, 2009.

[8] A. Kumar, J. Kaur, and P. Singh, "A new method for solving fully fuzzy linear programming problems," Applied Mathematical Modelling, vol. 35, no. 2, pp. 817-823, 2011.

[9] C. Jiang, X. Han, G. R. Liu, and G. P. Liu, "A nonlinear interval number programming method for uncertain optimization problems," European Journal of Operational Research, vol. 188, no. 1, pp. 1-13, 2008.

[10] J. Li, J. P. Xu, and M. S. Gen, "A class of multiobjective linear programming model with fuzzy random coefficients," Mathematical and Computer Modelling, vol. 44, no. 11-12, pp. 1097-1113, 2006. 
[11] Z. Wan, K. L. Teo, L. S. Kong, and C. Yang, "A class of mix design problems: formulation, solution methods and applications," The ANZIAM Journal, vol. 50, no. 4, pp. 455-474, 2009.

[12] Q. G. Lin, G. H. Huang, B. Bass, and X. S. Qin, "IFTEM: an interval-fuzzy two-stage stochastic optimization model for regional energy systems planning under uncertainty," Energy Policy, vol. 37, no. 3, pp. 868-878, 2009.

[13] X. S. Qin, G. H. Huang, G. M. Zeng, A. Chakma, and Y. F. Huang, "An interval-parameter fuzzy nonlinear optimization model for stream water quality management under uncertainty," European Journal of Operational Research, vol. 180, no. 3, pp. 1331-1357, 2007.

[14] I. Elishakoff and P. Colombi, "Combination of probabilistic and convex models of uncertainty when scarce knowledge is present on acoustic excitation parameters," Computer Methods in Applied Mechanics and Engineering, vol. 104, no. 2, pp. 187209, 1993.

[15] X. P. Du and A. Sudjianto, "Reliability-based design with the mixture of random and interval variables," in Proceedings of the ASME Design Engineering Technical Conferences and Computers and Information in Engineering Conference, pp. 55-62, Chicago, Ill, USA, September 2003.

[16] X. P. Du, "Interval reliability analysis," in Proceedings of the ASME International Design Engineering Technical Conferences and Computers and Information in Engineering Conference (IDETC '07), pp. 1103-1109, Las Vegas, Nev, USA, September 2007.

[17] C. Jiang, W. X. Li, X. Han et al., "Structural reliability analysis based on random distributions with interval parameters," Computers \& Structures, vol. 89, no. 23-24, pp. 2292-2302, 2011.

[18] C. Jiang, X. Han, W. X. Li, and Z. Zhang, "A hybrid reliability approach based on probability and interval for uncertain structures," Journal of Mechanical Design, vol. 134, no. 3, Article ID 031001, 11 pages, 2012.

[19] S. J. Zhang and Z. Wan, "Polymorphic uncertain nonlinear programming model and algorithm for maximizing the fatigue life of V-belt drive," Journal of Industrial and Management Optimization, vol. 8, no. 2, pp. 493-505, 2012.

[20] Z. Wan, S. J. Zhang, and K. L. Teo, “Two-step based sampling method for maximizing the capacity of V-belt driving in polymorphic uncertain environment," Proceedings of the Institution of Mechanical Engineers C, vol. 226, no. 1, pp. 177-191, 2012.

[21] Christer Carlsson and Robert Fullér, Fuzzy Reasoning in Decision Making and Optimization, Physica, Heidelberg, Germany, 2002.

[22] M. Liu, S. M. Lo, B. Q. Hu, and C. M. Zhao, "On the use of fuzzy synthetic evaluation and optimal classification for computing fire risk ranking of buildings," Neural Computing and Applications, vol. 18, pp. 643-652, 2009. 


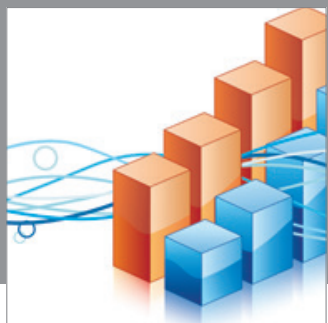

Advances in

Operations Research

mansans

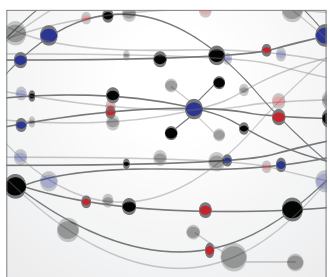

The Scientific World Journal
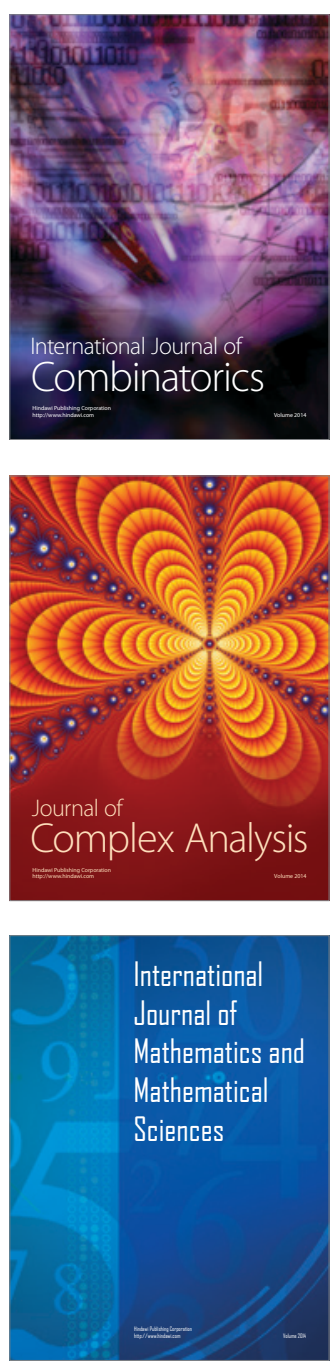
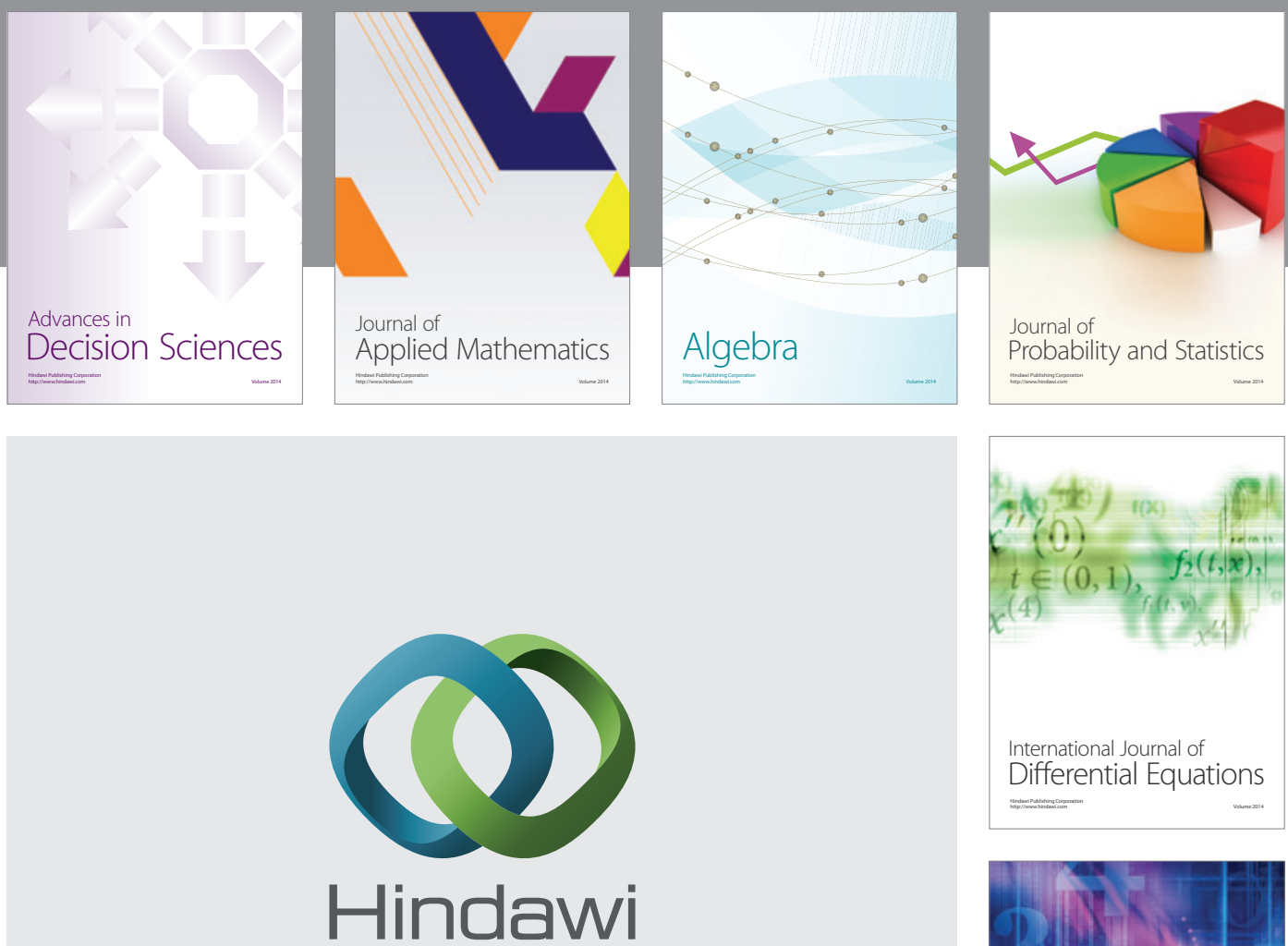

Submit your manuscripts at http://www.hindawi.com
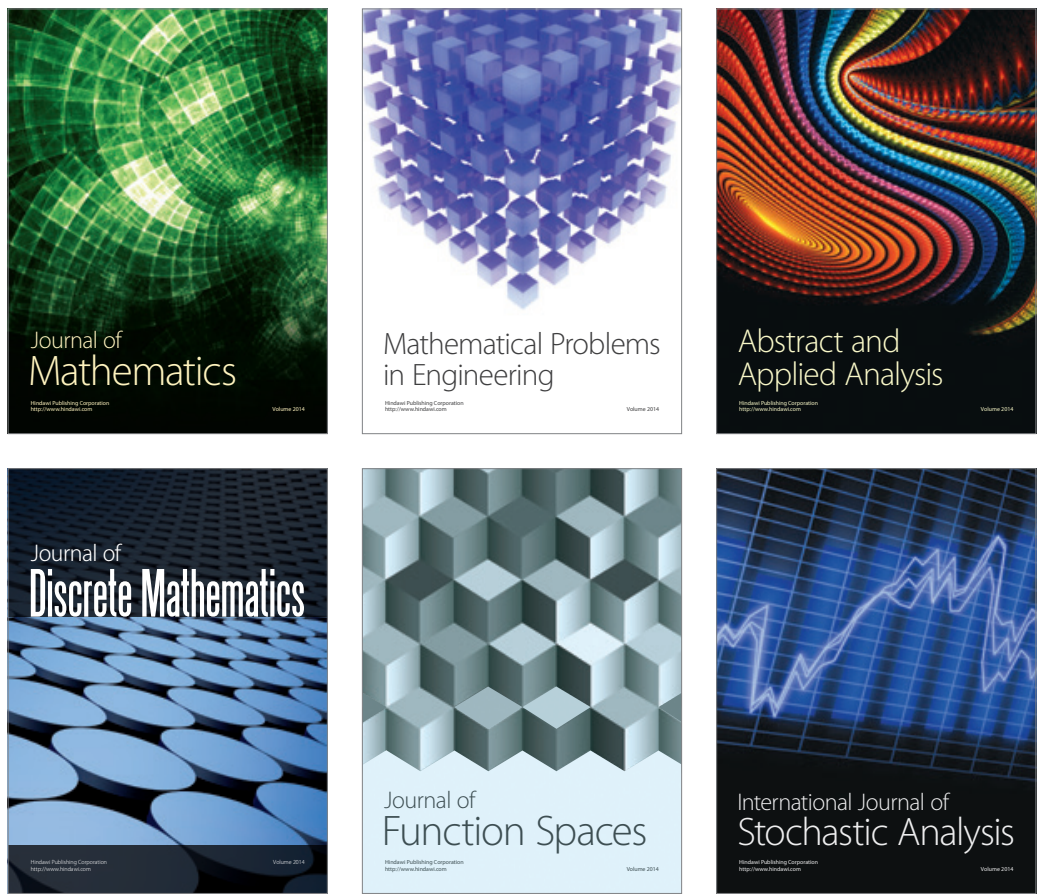

Journal of

Function Spaces

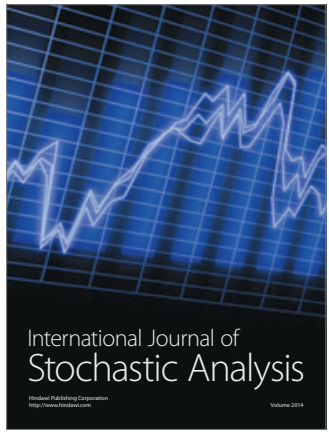

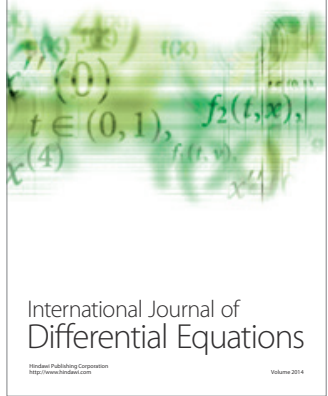
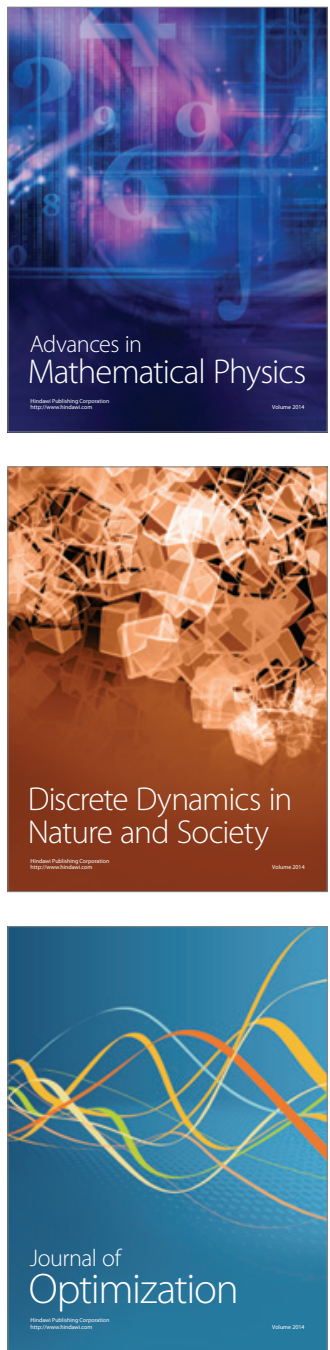